\title{
A new flight conflict detecting and avoiding method based on the missile characteristics
}

\author{
YuLiang Liu ${ }^{1, a^{*}}$, Jiaxuan $\mathrm{Xie}^{2, \mathrm{~b}}$ and $\mathrm{Ke} \mathrm{Li}{ }^{3, \mathrm{c}}$ \\ ${ }^{1}$ Digital Engineering Research Institute, Wuhan, China \\ ${ }^{2}$ Naval Equipment Research Institute, Beijing, China \\ ${ }^{3}$ Naval university of Engineering, Wuhan, China \\ a120049214@163.com
}

\begin{abstract}
Keywords: flight conflict; adjusting speed; adjusting direction; comprehensive adjustment
Abstract. In modern air combat, it is very important for the aircraft to detect the coming missile and avoid. In traditional flight conflict detection method, more attentions are paid on plane to plane. In this occasion, the plane is flying according to the planed routes. But for the missile, once the missile seeker finds the target, the planed routes must be changed to destroy the target. Moreover, the destroying point is not the current target location, but the future location. So the traditional flight conflict detection method is not fit for the conflict between the plane and missile. Above all, A new flight conflict detecting and avoiding method based on the missile characteristics is proposed in the paper.
\end{abstract}

\section{Flight interval}

Missile searching sector

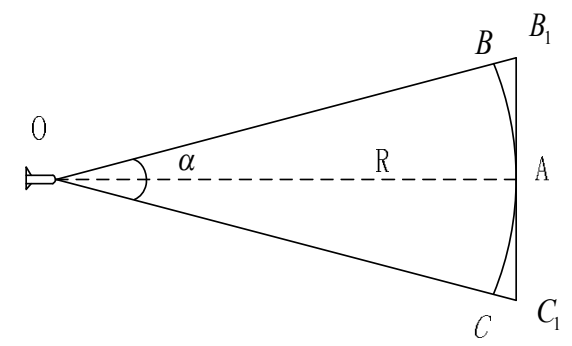

Fig.1 Missile searching sector

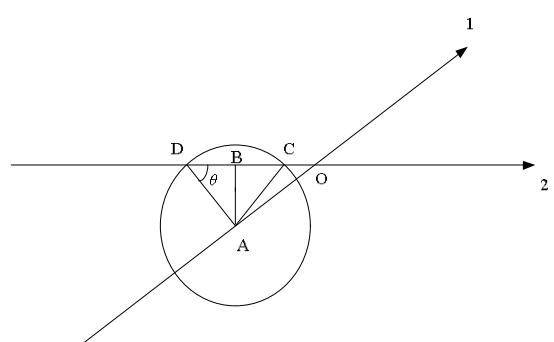

Fig.2 Air route conflict between two planes

The missile seeker is like a sector when searching plane, the searching radius is $O B=O C=O A=R$, the searching angle is $\alpha$, showed in figure 1. In order to enlarge the searching area, the missile searching sector is extended to a isosceles triangle ${ }^{[1]}$. The line $B_{1} C_{1}$ is tangent to arc $B A C$ on the point A. The length of two edges is (showed in figure 3 ) :

$$
B E_{1}=B D_{1}=R / \cos \frac{\alpha}{2}
$$

\section{Safe airspace interval}

The safe airspace interval is the least distance between different planes to avoid the flight conflict. In traditional flight conflict detecting method, the conflict is happened between the plane to plane ${ }^{[2]}$. And the dangerous areas in this conflict detecting are several the same circles, showed in figure 2.

In figure 2, point $A$ is the plane location on route $1 . A D=A C$ is the safe di stance. $A B=A C \times \sin \theta$ is the distance between the plane on route 1 to the plane on route 2 . If $A B \leq A C$, the two planes may collide. But in the conflict between the missile and aircraft, the searching area is like a sector and the radius is much more larger than the cinflict area radius $R_{f}$. The searching angle is $\alpha$, the serching radius is $R$. So a more efficient detecting method must be proposed. 


\section{Conflict description}

\section{Flight route analysis}

Although the missile and plane are not on the same surface in the realistic air combat, the problem is simplized in the paper. The missile velocity is broken down ${ }^{[3]}$. The projection of the plane and missile velocity on the horizontal plane is analyzed instead of the real flying velocity, showed in figure 3.

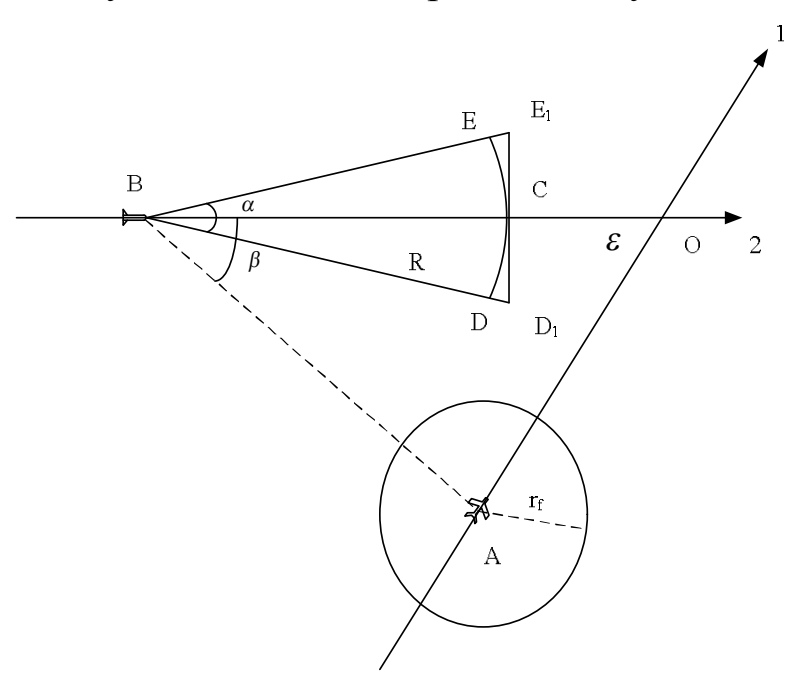

Fig. 3 route conflict between plane and missile

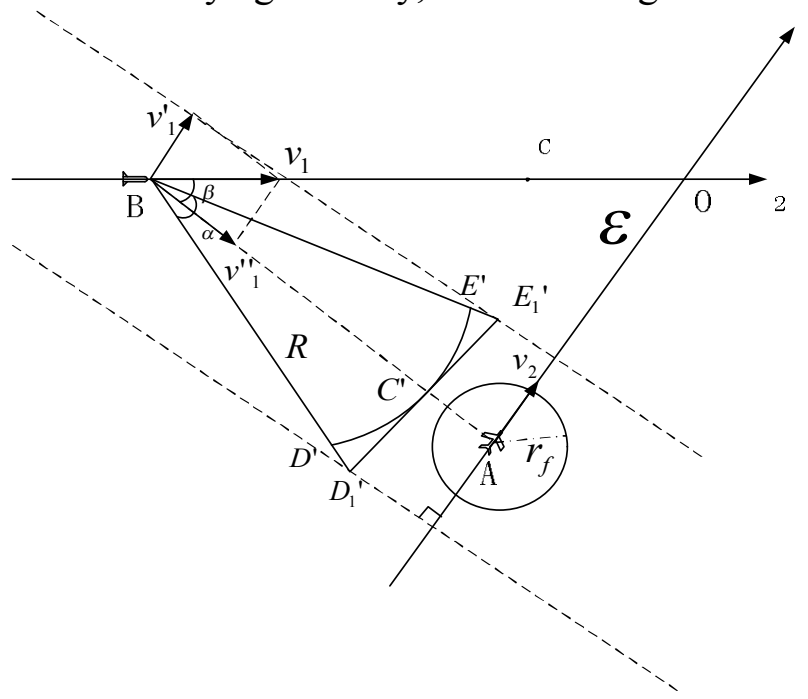

Fig. 4 route conflict after velocity is broken down

The missile is flying from point $\mathrm{B}$ with the velocity $v_{1}$ along route 1 . After time $t$, the missile reaches point $O$. The angle between the missile flying route and the line from the missile to plane is $\beta$. The plane is flying from point $\mathrm{A}$ with the velocity $v_{2}$ along route 2 . After time $t$, the plane reaches point $O$. The safe distance of the plane is $R_{f}$. The angle of the two routes is $\varepsilon$.

The distance from missile to point $O$ is $B O=v_{1} \times t$.

The distance from missile searching sector vertex to point $O$ is $C O=R-v_{1} \times t$.

The straight distance on the horizontal plane from the missile to plane is :

\section{Conflict analysis}

$$
A B=\sqrt{A O^{2}+B O^{2}-2 A O \cdot B O \cdot \cos \varepsilon}
$$

When the missile searching sector reaches the plane safe area, the conflict happened, which can be judged by the angle between the missile flying route and the line from the missile to plane ${ }^{[4-5]}$.

After time $t$, both missile and plane reach point $O$, so the missile velocity is broken down, showed in figure 4.

If the plane did not leave the searching area of missile seeker within the limited time, still stayed in the triangle $B D_{1}{ }^{\prime} E_{1}{ }^{\prime}$, the plane is judged caught by the missile and the conflict happened.

The limited time is :

$$
t_{1}=A C_{1}-r_{f} / v_{1}^{\prime \prime}=\frac{A B-r_{f}-R}{v_{1}^{\prime \prime}}=\frac{\sqrt{A O^{2}+B O^{2}-2 A O \cdot B O \cdot \cos \varepsilon}-R-r_{f}}{v_{1}^{\prime \prime}}
$$

If $\left|v_{2}-v_{1}\right| t_{1} \leq C^{\prime} E_{1}^{\prime}$, the conflict between the plane and missile happened.

\section{Conflict avoid}

\section{Adjusting speed}

As one way to avoid the conflict, the plane speed along the route 1 can be adjusted. If the plane must go through the searching area of the missile, the velocity $v_{2}$ of plane A can be adjusted to avoid the conflict. And the adjusting condition is :

$$
\Delta v_{2} t_{1} \geq C^{\prime} E_{1}^{\prime}
$$


If the plane tried to pass in front of the missile searching area, the speed variable $\Delta v_{2}$ must be bigger than a certain number, $\Delta v_{2} \geq v_{m}$. If the plane tried to pass from the back of the missile searching area, the speed variable $\Delta v_{2}$ must be smaller than a certain number, $\Delta v_{2} \leq v_{n}$, which means the conflict has been resolved. Of course, the plane must be in the range of the plane performance parameter.

\section{Adjusting direction}

As another way to avoid the conflict, the plane flying direction along the route 1 can be also adjusted. The former angle between the missile and the plane is $\varepsilon$, once the direction changes, the angle becomes $\varepsilon+\Delta \varepsilon$.

For the missile must fly along the scheduled route, its direction remains the same. So only the

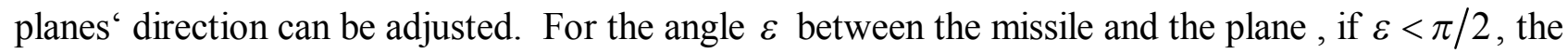
plane chooses to fly from the front of the missile. If $\varepsilon>\pi / 2$, the plane chooses to fly from the back of the missile.

As is showed in figure 5, the least distance from the turning point to the missile route must be bigger than the sum of the searching radius and the plane safe distance.

$$
C E_{1}=C D_{1}<d-r_{f}
$$
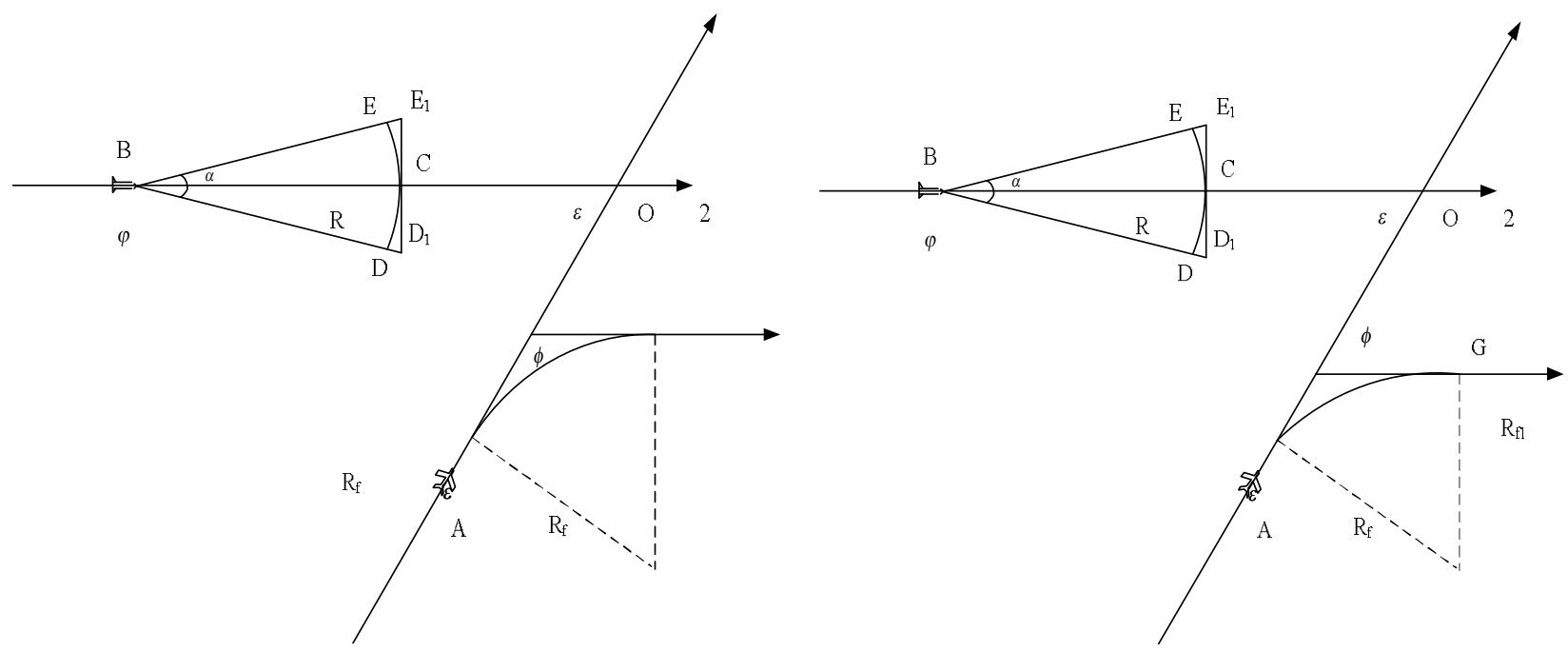

Fig.5 flying routes after direction adjusted

Fig.6 flying routes after comprehensive adjustment

At this time, the plane can pass through the side of the missile route and couldn't be detected. The plane changing angle is $\varphi$, the changing radius is $R_{f}$. In the paper, the plane speed remains the same when changing direction.

$$
\begin{gathered}
A A_{1}=2 \times \pi \times R_{f} \times \frac{\pi-\varphi}{2 \times \pi}=R_{f} \times(\pi-\varphi) \\
\varphi<\pi-\varepsilon
\end{gathered}
$$

The changing diretion time $t_{1}$ must be shorter than the time $t$ that the missile searching sector reaches the point $O$. So:

$$
t_{1}=\frac{R_{f} \times(\pi-\varphi)}{v_{2}} \leq t
$$

If the above equation is satisfied, the conflict is resolved. Of course, the plane must be in the range of the plane performance parameter.

\section{Comprehensive Adjustment}

After adjusting the speed or diretion, in some occasion, the conflict can also be happened. So sometimes, the speed and direction must be adjusted comprehensively. Once the conflict still happens after speed adjusted, the plane flying direction must also be adjusted and changes the route. 
When the plane changes direction, the angular velocity is stationary. So the changing radius is in proportion to the linear velocity. If the speed is adjusted, the radius also changed. The arc length that plane passed is :

$$
A D=\int_{R_{f 1}}^{R_{f}} 2 \pi r \times \frac{\varphi}{2 \pi} d r
$$

The changing diretion time $t_{1}$ must be shorter than the time ${ }^{t}$ that the missile searching sector reaches the point $O$. So:

$$
t_{1}=\int_{R_{f 1}}^{R_{f}} \frac{r \times(\pi-\varphi)}{v_{2}} d r \leq t
$$

If the above equation is satisfied, the conflict is resolved. Of course, the plane must be in the range of the plane performance parameter.

\section{Simulation analysis}

The current plane flying speed is $200 \mathrm{~m} / \mathrm{s}$, the upper bound of the flying speed is $400 \mathrm{~m} / \mathrm{s}$, the lower bound of the flying speed is $100 \mathrm{~m} / \mathrm{s}$, the acceleration is $250 \mathrm{~m} / \mathrm{s}$, the missile speed is $800 \mathrm{~m} / \mathrm{s}$, the radius of missile searching sector is $600 \mathrm{~m}$, the angle of searching sector is $8^{\circ}$. In this occasion, the plane is the changing variable. So in this simulation, the angle $\varepsilon$ between the plane and the missile is random, and $0 \leq \varepsilon<\pi$. The distance from missile to the plane point in advance are respectively $1000 \mathrm{~m}, 1500 \mathrm{~m}, 2000 \mathrm{~m}, 2500 \mathrm{~m}, 3000 \mathrm{~m}, 3500 \mathrm{~m}$ and $4000 \mathrm{~m}$. After 10000 times simulation, the conflict result could be analyzed. Finally, the conflict happening ratio is showed in figure 7:

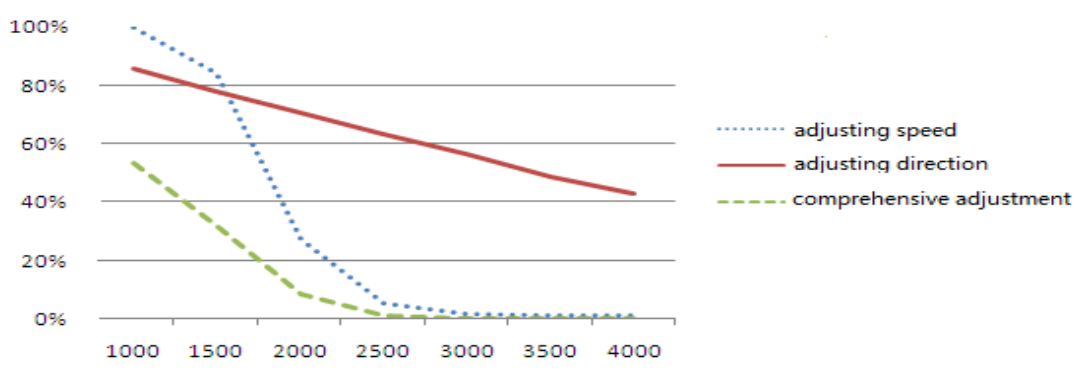

Fig.7 simulation result

From the figure 7, after comprehensive adjustment on speed and direction, the conflict ratio is much lower than others and the plane can avoid the missile. Meanwhile, the result of comprehensive adjustment is better. The speed and direction are both studied in the paper. With the analysis of the simulation, the conflict avoiding method proposed above could efficiently decrease the conflict ratio.

\section{References}

- [1] Lu Jianbin, XIAO Hui, XI Zemin, ZHANG Mingmin. Phased array radar resource management: task scheduling and performance evaluation, Journal of Computational Information Systems, 9(3), pp 1131-1138, 2013.

- [2] Arnon G, Israel C . Anomaly detection based on an iterative local statistics approach[J]. Signal Processing, 2004, (84): 1225-1229.

- [3] The Cooperative Engagement Capability[J]. Johns Hopkins APL Technical Digest. 1995,16(4):9 12.

- [4] Caron Y, Makris, Vincent N. A method for detecting artificial objects in natural environments[J]. International conference on pattern recognition (PR 2002), Canada, 2002: 600-603. 
- [5] S Coleri, M Ergen, et al. Channel Estimation techniques based on pilot arrangement in OFDM Systems[J]. IEEE Transactions on Broadcasting,2002,48(3):223 229. 